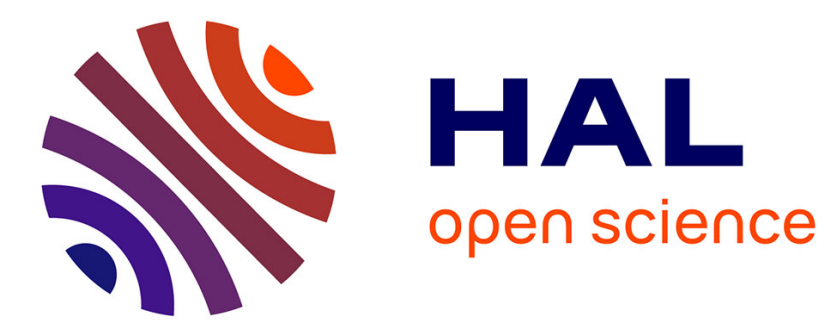

\title{
Étude de l'impact d'une perturbation électromagnétique sur le fonctionnement de composants logiques électroniques
}

Catherine Maréchal, Marco Klingler, Marc Heddebaut, Bernard Demoulin

\section{To cite this version:}

Catherine Maréchal, Marco Klingler, Marc Heddebaut, Bernard Demoulin. Étude de l'impact d'une perturbation électromagnétique sur le fonctionnement de composants logiques électroniques. Journal de Physique III, 1995, 5 (6), pp.743-756. 10.1051/jp3:1995158 . jpa-00249343

HAL Id: jpa-00249343

https://hal.science/jpa-00249343

Submitted on 1 Jan 1995

HAL is a multi-disciplinary open access archive for the deposit and dissemination of scientific research documents, whether they are published or not. The documents may come from teaching and research institutions in France or abroad, or from public or private research centers.
L'archive ouverte pluridisciplinaire HAL, est destinée au dépôt et à la diffusion de documents scientifiques de niveau recherche, publiés ou non, émanant des établissements d'enseignement et de recherche français ou étrangers, des laboratoires publics ou privés. 
Classification

Physics Abstracts

$06.30 \mathrm{~F}-06.70 \mathrm{E}$

\title{
Étude de l'impact d'une perturbation électromagnétique sur le fonctionnement de composants logiques électroniques
}

\author{
Catherine Maréchal $\left({ }^{\mathbf{1}}\right)$, Marco Klingler $\left({ }^{\mathbf{1}}\right)$, Marc Heddebaut $\left({ }^{1}\right)$ et Bernard Demoulin $\left({ }^{2}\right)$ \\ ( ${ }^{1}$ INRETS (Institut National de Recherche sur les Transports et leur Sécurité) LÉOST (Labo- \\ ratoire Électronique Ondes et Signaux pour les Transports), 20 rue Élisée Reclus, 59650 Ville- \\ neuve d'Ascq, France \\ $\left({ }^{2}\right)$ USTL-LRPE (Université des Sciences et Technologies de Lille - Laboratoire de Radio- \\ Propagation et Électronique), 59650 Villeneuve d'Ascq, France
}

(Reçu le 15 juin 1994, accepté le 22 février 1995)

\begin{abstract}
Résumé. - Nous nous intéressons dans cet article à la susceptibilité électromagnétique de composants électroniques logiques. Plusieurs techniques de réalisation de ces composants sont passées en revue. La première partie est consacrée à l'étude d'un dispositif sous test simple composé de deux portes NAND connectées en cascade. La simplicité de ce dispositif facilite la compréhension du processus de perturbation. La deuxième partie est consacrée à l'étude d'une carte électronique inspirée d'une carte réalisant une fonction de sécurité sur le métro automatique de Lille. Ce travail s'apparente à l'étude d'un cas réel et pratique. La sûreté de fonctionnement est ici fondée sur l'utilisation d'une redondance matérielle de la fonction suivie d'un comparateur de signaux.
\end{abstract}

\begin{abstract}
This paper deals with the susceptibility of digital electronic components depending on the components' technology. The first part of this paper will present the results obtained on a simple circuit under test composed of two digital gates interconnected together. The second part will deal with a practical case study of a printed circuit board representing an electronic safety function on the automatic metro of Lille based on an active material redundancy.
\end{abstract}

\section{Introduction}

La compatibilité électromagnétique (CEM) est l'aptitude d'un équipement ou d'un système à fonctionner de façon satisfaisante dans son environnement électromagnétique sans produire lui même de perturbations électromagnétiques intolérables pour quoi que ce soit dans cet environnement. Des études montrent que la mise aux normes CEM d'un système coûte d'autant plus chère qu'elle est effectuée tardivement dans le processus de fabrication. Il importe donc de prendre en compte la CEM des produits dès leur conception.

Nous nous intéressons dans cet article à la sensibilité électromagnétique des systèmes électroniques. Les perturbations produites au niveau d'un dispositif électronique se propagent par 
conduction au niveau des sous-dispositifs pour arriver finalement aux étages d'entrée/sortie des composants électroniques. Le comportement de ces composants vis à vis des perturbations intervient donc sur la sensibilité globale du dispositif électronique. C'est pourquoi nous étudions la susceptibilité des composants logiques électroniques selon leur technologie. Nous analysons également la sûreté de fonctionnement d'un système redondant actif soumis à une perturbation électromagnétique, les composants des voies du système étant de technologies différentes. L'objectif de cette étude est de montrer l'importance du choix, en termes de CEM, de la technologie des composants lors de la conception d'un système et de fournir, à terme, des informations pratiques pour aider les concepteurs et les ingénieurs chargés de la sécurité à choisir une technologie de composants.

\section{1. Étude d'un dispositif sous test simple}

1.1. Introduction. - Les travaux effectués sur des dispositifs sous test simples constitués de composants électroniques logiques ont révélé trois types de défauts sur les signaux selon le niveau du perturbateur (Fig. 1) [1]. Des avances ou des retards de signaux par rapport aux instants idéaux de commutation apparaissent pour des amplitudes faibles du perturbateur [2-4]. Aux amplitudes un peu plus élevées, des distorsions du front de transit logique ou pseudocommutations sont observées [4]. Enfin pour un couplage fort, les états logiques statiques sont perturbés [5-7].

Notre travail consiste à caractériser les défauts qui arrivent sur les états statiques pour des perturbations de forte puissance. Il complète l'étude statistique réalisée par Coudoro [4] sur les retards ou avances de fronts logiques.

Pour des fréquences élevées de perturbations, des phénomènes de redressement apparaissent dans les jonctions PN. Les caractéristiques statiques des diodes ou transistors bipolaires sont modifiées, car la valeur moyenne du signal redressé change les points de polarisation [8]. D'autre part, en première approche, on peut considérer qu'au delà de sa fréquence maximale de travail, le composant joue un rôle de filtre passe-bas. Pour des amplitudes élevées d'agres-

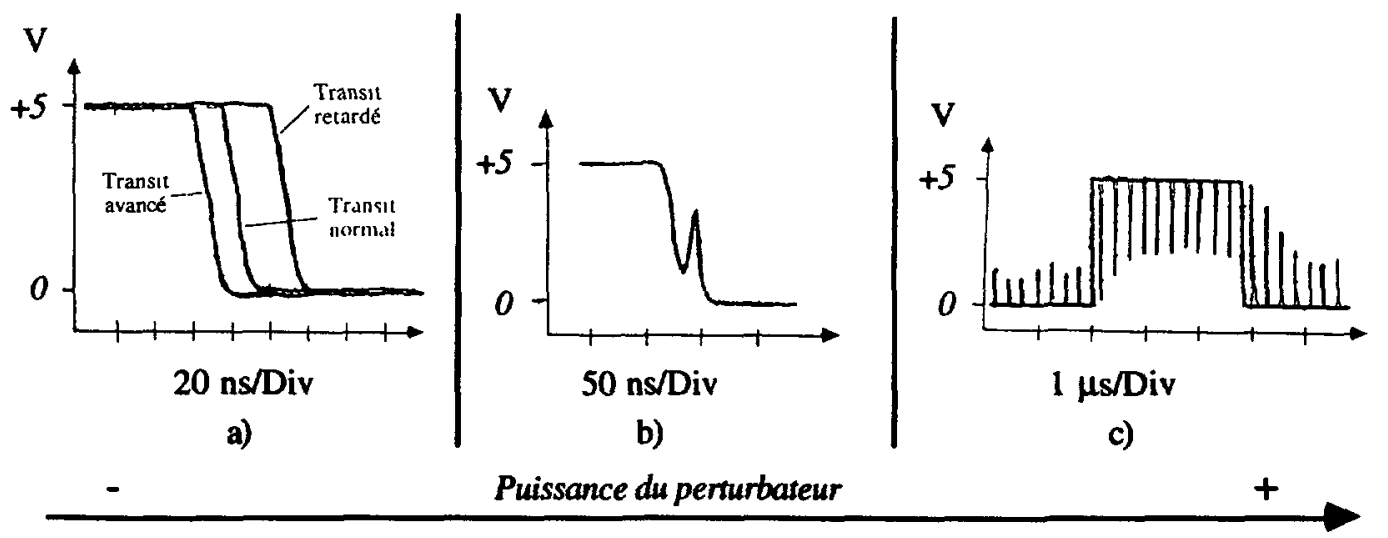

Fig. 1. - Les différents types de défauts observés sur des dispositifs sous test simples selon la puissance du perturbateur : a) Défauts du type avance ou retard de front, b) Pseudo-commutation, c) Défauts statiques.

[The different types of faults observed on simple circuits under test depending on the power of the RF disturbance a) Timing faults, b) Pseudo-commutation, c) Static faults.] 
sions électromagnétiques, les signaux en entrée des composants sont redressés par les diodes de protection. Si ces signaux ont une fréquence élevée par rapport à la fréquence maximale de travail des composants, leur valeur moyenne est détectée par les portes. La détection-filtrage du signal redressé génère une tension de polarisation supplémentaire qui se superpose à la tension de polarisation nominale. Cette polarisation supplémentaire décale donc les niveaux de fonctionnement nominaux des composants et notamment le seuil de commutation (Réf. [7], pp. 86 à 87). Les outils réalisés au laboratoire et présentés dans cet article - outil statistique et visualisation des extremums de tension du signal perturbé- révèlent l'impact de ces décalages sur les signaux en sortie des composants.

1.2. DesCription DU Dispositif SOUS TEST ET DU BANC DE MESUREs. - Les études précédentes ont montré que les capacités de découplage suffisent à protéger les signaux d'alimentation contre des perturbations électromagnétiques [5]. Nous nous intéressons donc à la perturbation des étages d'entrée/sortie des composants.

Le dispositif sous test initial est constitué de deux portes inverseuses montées en cascade et de technologie identique (Fig. 2a). La mise en parallèle de deux dispositifs sous test permet de tester deux technologies simultanément et de simuler un système redondant simple (Fig. 2b). La ligne de transmission qui relie les deux inverseurs est soumise à un champ électromagnétique généré par une cellule de type "stripline" dans laquelle se propage une onde électromagnétique plane (Fig. 2c). Le banc de mesures est entièrement automatisé.

Lors de l'étude statistique, l'information qui transite sur le système est un signal carré de fréquence $1 \mathrm{MHz}$. Les signaux sont enregistrés pour des fréquences de perturbateur variant de $15 \mathrm{MHz}$ à $95 \mathrm{MHz}$ par pas de $10 \mathrm{MHz}$ et une amplitude variant de $50 \mathrm{~V} / \mathrm{m}$ à $450 \mathrm{~V} / \mathrm{m}$ par pas de $50 \mathrm{~V} / \mathrm{m}$. Une fois tous les signaux acquis, ils sont traités à l'aide de l'outil statistique.

Lors de la visualisation des extremums de tension entre lesquels les signaux de sortie varient,

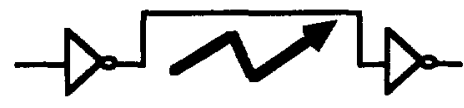

a)

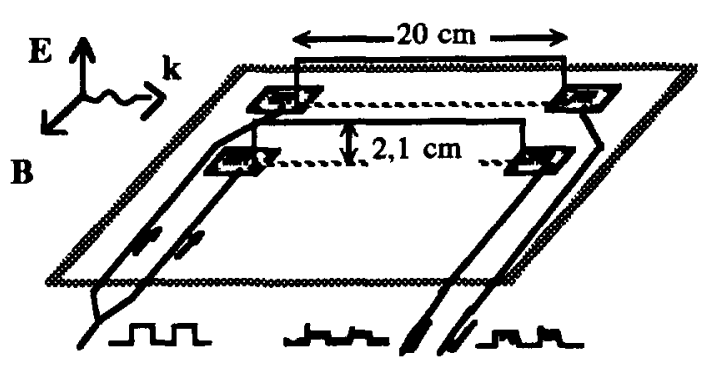

b)

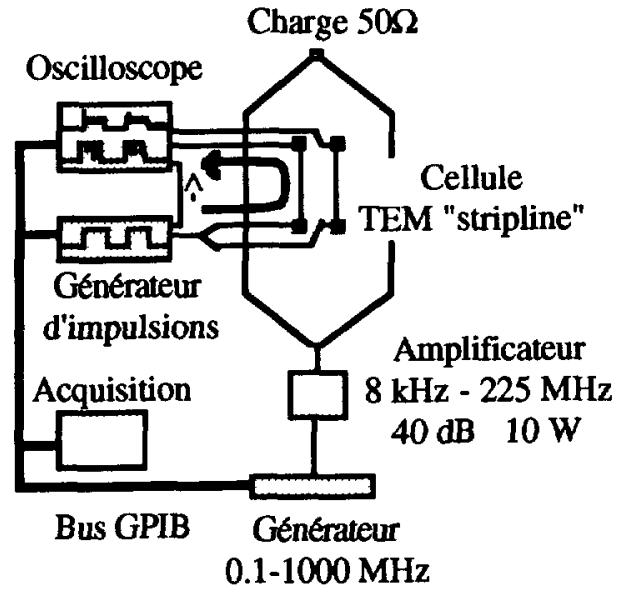

c)

Fig. 2. - a) Le dispositif sous test initial, b) Mise en parallèle de deux dispositifs sous test, c) Le banc de mesures.

[a) The initial circuit under test, b) Two circuits under test in parallel, c) The test bench.] 
l'entrée du dispositif sous test est constamment à l'état bas ou à l'état haut. La fréquence du champ électromagnétique perturbateur varie de $15 \mathrm{MHz}$ à $200 \mathrm{MHz}$ par pas de $10 \mathrm{MHz}$ et l'amplitude de $0 \mathrm{~V} / \mathrm{m}$ à une valeur approximative de $900 \mathrm{~V} / \mathrm{m}$.

\subsection{LES OUTILS UTILISÉS POUR ÉTUDIER LE DISPOSITIF SOUS TEST SIMPLE}

1.3.1. Principe de l'Étude statistique. - Un défaut est l'écart entre la caractéristique d'une entité et la caractéristique voulue, cet écart dépassant des limites d'acceptabilité. En ce qui concerne les niveaux de composants logiques, les limites d'acceptabilité sont les tensions de seuil données par les constructeurs. Le principe de l'étude statistique est donc de comparer, échantillon par échantillon, le signal perturbé à un signal non perturbé dit de référence (Fig. 3 ). Nous déterminons trois états possibles. L'état bas si le signal est en dessous de la tension de

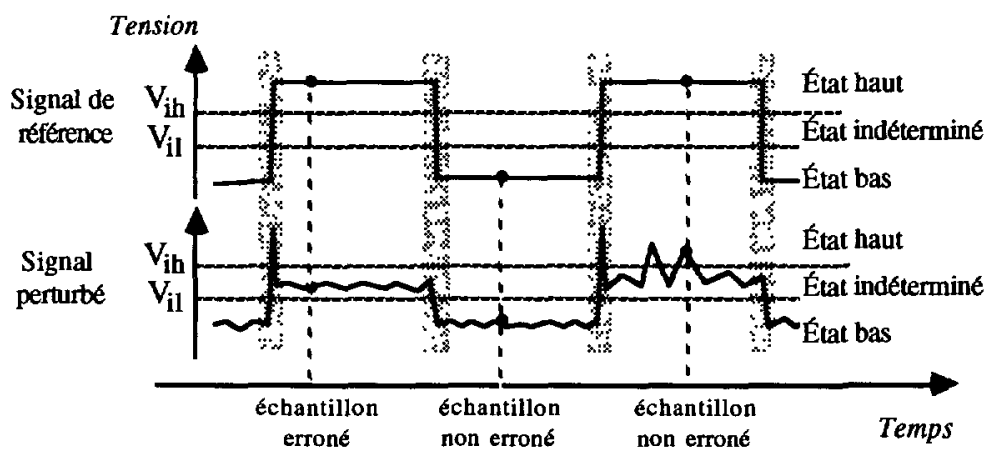

Fig. 3. - Principe de l'étude statistique. Le signal perturbé est comparé au signal de référence. Si un échantillon n'est pas dans le même état que l'échantillon de référence correspondant, il est considéré comme erroné.

[Principle of the statistical study. The disturbed signal is compared to the reference signal. If a sample is not in the same state as the corresponding reference sample, it is considered as erroneous.]

seuil niveau bas $V_{1}$, l'état haut si le signal est au-dessus de la tension de seuil $V_{\text {lh }}$ et l'état indéterminé. Si à un instant donné, l'échantillon du signal perturbé ne se trouve pas dans le même état que l'échantillon du signal de référence, celui-ci est considéré comme erroné. L'état indéterminé est ainsi considéré comme un état erroné. Une fenêtre de $20 \mathrm{~ns}$ est définie autour de chaque front pour limiter l'étude aux états statiques. Nous présentons dans cet article la caractéristique la plus représentative, à savoir le pourcentage de temps durant lequel les niveaux sont perturbés.

1.3.2. Méthode de mesures pour visualiser les extremums de tension entre lesquels les signaux perturbés varient. - Lorsque la fréquence du signal modulant est très inférieure à la fréquence de la porteuse, le régime modulé en amplitude est localement équivalent à un régime harmonique (Fig. 4). En utilisant une rampe comme signal modulant, nous obtenons donc un signal harmonique dont l'amplitude varie linéairement avec le temps (Fig. 5). En entrée du dispositif sous test, nous imposons un état logique bas ou haut et nous soumettons le système au perturbateur. Nous visualisons les courbes $X Y$ obtenues en mesurant la rampe du signal de modulation sur la voie $X$, et en mesurant la sortie du dispositif sous test sur la voie $Y$ de l'oscilloscope. La signification physique des courbes obtenues est expliquée figure 6. 


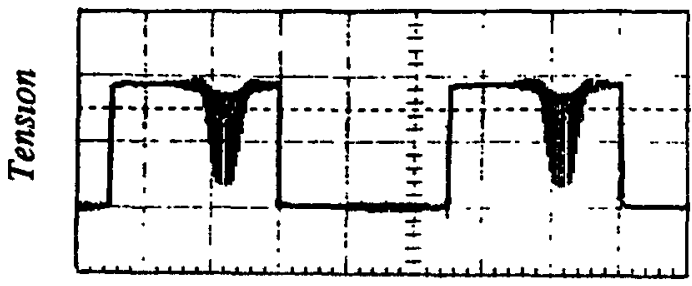

Temps

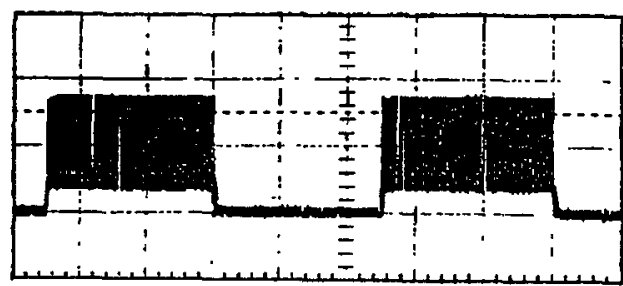

Temps

a)

b)

Fig. 4. - Composants de technologie TTL-ALS, fréquence du perturbateur $65 \mathrm{MHz}$. a) Régime modulé en amplitude, $E=88 \mathrm{~V} / \mathrm{m}, m=100 \%$; b) Régime harmonique pur, $E=176 \mathrm{~V} / \mathrm{m}$. Lorsque localement l'amplitude du champ électromagnétique modulé en amplitude est maximale et égale à 176 $\mathrm{V} / \mathrm{m}$, la perturbation observée est identique à celle produite par un champ harmonique d'amplitude $176 \mathrm{~V} / \mathrm{m}$.

[Components of TTL-ALS technology, Frequency of the RF disturbance $65 \mathrm{MHz}$. a) Amplitude modulated disturbance, $E=88 \mathrm{~V} / \mathrm{m}, m=100 \%$; b) Harmonic disturbance, $E=176 \mathrm{~V} / \mathrm{m}$. When the local amplitude of the modulated field is equal to $176 \mathrm{~V} / \mathrm{m}$, the faults are the same as the faults due to the harmonic disturbance of $176 \mathrm{~V} / \mathrm{m}$.]

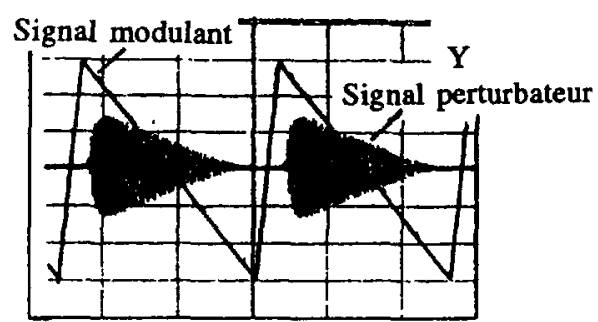

Temps

a)

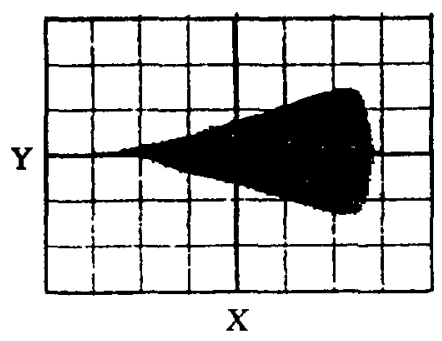

b)

Fig. 5. - Signal modulé en amplitude utilisé pour visualiser l'excursion des défauts selon l'amplitude du champ électromagnétique perturbateur. a) Courbes temporelles, b) Courbe de Lissajous.

[Amplitude modulated signal used to visualize the excursion of the faults depending on the amplitude of the electromagnetic field. a) Time domain, b) $X Y$ curve.]

1.4. IMPACT DE LA PERTURBATION SUR L'ALIMENTATION DES COMPOSANTS. - Lors de nos expériences, toutes les précautions sont prises pour éviter la perturbation des signaux d'alimentation. La tension de $9 \mathrm{~V}$ fournie par une pile plate est ramenée sur les éléments sous test au moyen de fils tressés collés au plan de masse. Le régulateur de tension est placé le plus près possible du composant à alimenter. Des capacités de découplage sont utilisées.

Cependant, les graphes visualisant l'excursion des défauts selon l'amplitude du champ électromagnétique semblent montrer que, contrairement aux composants de technologie CMOS, l'alimentation des composants de technologie TTL est perturbée pour des amplitudes élevées (Fig. 7a). En effet, pour cette technologie, l'excursion des défauts sur niveaux hauts (Fig. 7a), suit celle de la perturbation ( $F$ ig. $5 b$ ). En ajoutant sur les cartes imprimées des traversées afin 


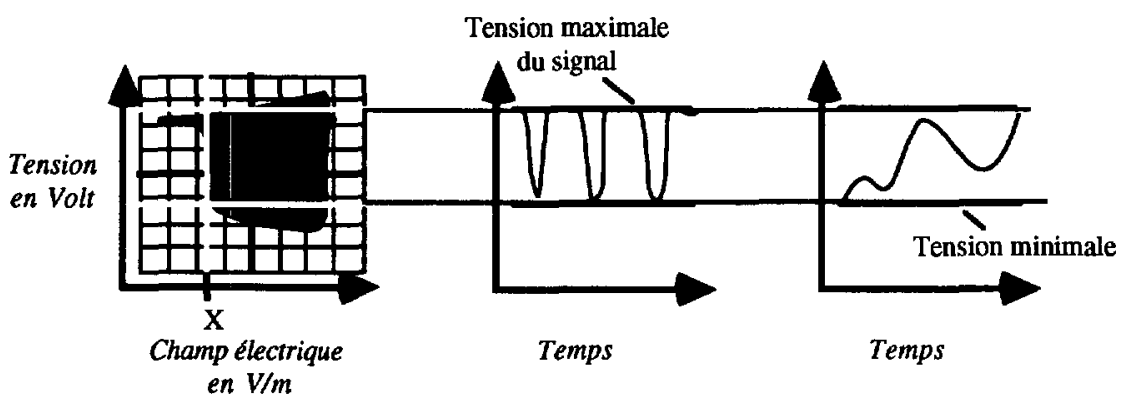

Fig. 6. - Les graphes donnant l'excursion des défauts selon l'amplitude du champ électromagnétique ne permettent pas de connaitre la variation temporelle des signaux. Ces graphes donnent les tensions minimales et maximales entre lesquelles le signal perturbé varie.

[The graphs give the minimal and maximal voltage between which the disturbed signal vary. The time domain of the signal is not visible.]

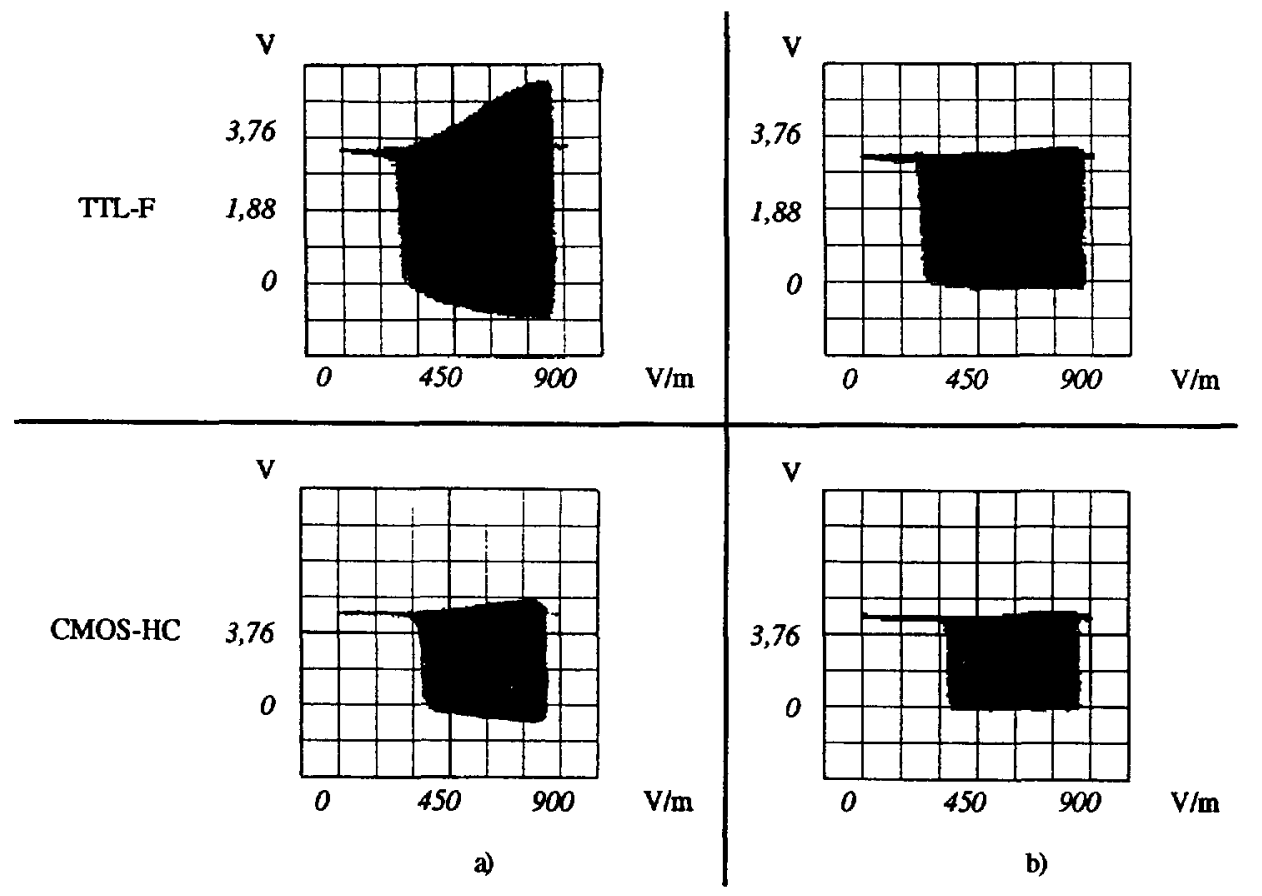

Fig. 7. - Excursion des défauts pour les technologies TTL-F et CMOS-HC. Fréquence du perturbateur : $95 \mathrm{MHz}$. a) Une seule traversée relie la piste de masse côté composants au plan de référence, b) Trois traversées relient la piste de masse au plan de référence.

[Excursion of the faults for the technologies TTL-F and CMOS-HC. Frequency of the perturbation of $95 \mathrm{MHz}$. a) A single crossing connects the ground track on the components'side to the ground plan, b) Three crossings connect the ground track to the ground plan.]

de relier directement la masse côté composants au plan de référence (et par conséquent à la masse de la cellule), l'excursion des défauts observées en sortie des composants TTL ne suit plus celle de la perturbation (Fig. 7b). On en déduit ainsi que la perturbation s'achemine par 


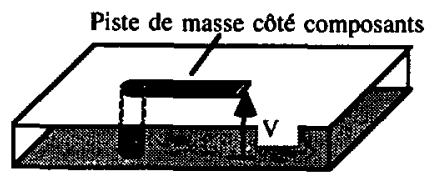

a)

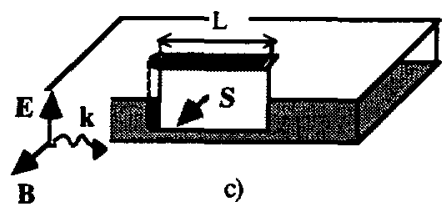

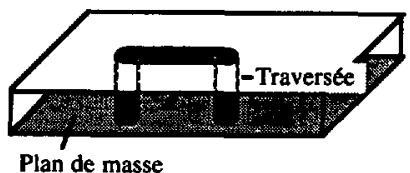

b)

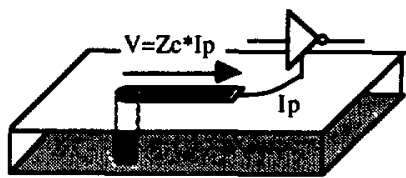

d)

Fig. 8. - a) Lorsqu'une seule traversée connecte la piste de masse au plan de référence, une tension parasite apparaît. b) La tension parasite disparaît si une traversée est ajoutée. c) Première cause susceptible de contribuer à la création de cette tension parasite : le champ électromagnétique crée par couplage une force électromotrice. d) Seconde cause : les appels de courant qui apparaissent sur l'alimentation à la même fréquence que le perturbateur, génèrent une tension dans l'inductance de la piste de masse.

(a) When a single crossing connects the ground track to the ground plan, a voltage appears on the ground track. b) The voltage disappears if a crossing is added. c) First cause: a direct disturbance of the electromagnetic field. d) Second cause: the voltage is also due to the supply current flowing through the impedance of the ground track.]

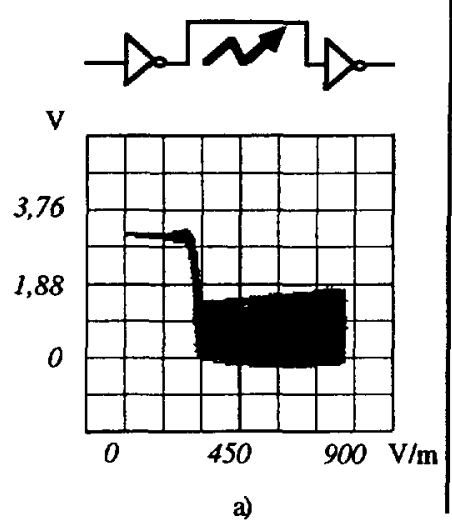

a)
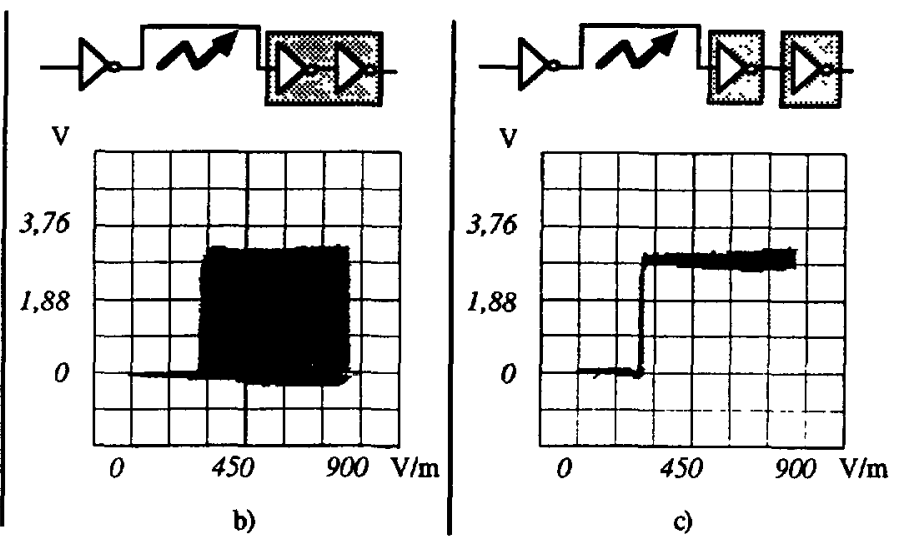

Fig. 9. - Composants de technologie TTL-LS, Perturbateur de fréquence $95 \mathrm{MHz}$. De part les appels de courant de l'alimentation du boitier de sortie, l'impact de la perturbation (a) sur une porte inverseuse n'est pas le même selon que la porte se situe sur le boîtier de sortie (b) ou sur un autre boîtier (c). [Components of TTL-LS technology, Frequency of the perturbation of $95 \mathrm{MHz}$. Because of the supply current the graph b) and c) are not the same.]

les circuits d'alimentation pour des perturbations élevées.

Le fait que l'alimentation soit perturbée lorsqu'une seule traversée connecte la masse côté composants au plan de référence, est sans doute dû à l'apparition d'une différence de potentiel sur la piste de référence (Fig. 8a) qui disparaît si on ajoute une traversée (Fig. 8b). Deux causes sont susceptibles de contribuer à l'apparition de cette tension parasite: 
i) La première cause est l'apparition d'une force électromotrice due au couplage du champ électromagnétique sur la surface délimitée par la piste de masse côté composants (Fig. 8c).

ii) La seconde cause est liée au fonctionnement interne des portes. Lors du fonctionnement normal d'une porte logique, la commutation du composant est accompagnée d'un fort appel de courant fourni par l'alimentation et assimilable à une impulsion. Lorsque le signal d'entrée est perturbé par un signal haute fréquence, les défauts peuvent être perçus par le composant logique comme des transitions logiques. L'alimentation fournit alors des impulsions de courant dont la fréquence d'apparition est identique à la fréquence du perturbateur. Le passage des courants de retour dans l'inductance de la piste engendre la tension parasite (Fig. 8d). Les traversées ajoutées notamment sur le circuit imprimé de l'inverseur de sortie permettent d'écouler ces courants de retour par un chemin de basse impédance.

Du fait des appels de courant sur l'alimentation du boîtier de sortie, l'impact des perturbations sur une porte inverseuse située sur le boîtier de sortie peut différer de l'impact des perturbations sur une porte inverseuse située sur un second boîtier (Fig. 9).

\subsection{Comparaison de la SUSCEPTibilité des technologies en RÉgime harmonique}

Nous avons étudié cinq familles de la technologie TTL (LS, ALS, AS, F et S) et deux familles de la technologie CMOS ( $\mathrm{HC}$ et $\mathrm{HCU}$ ). Les statistiques révèlent un comportement différent des composants selon qu'il s'agit de technologies TTL ou CMOS. Les composants de technologie TTL sont perturbés uniquement sur les niveaux hauts tandis que les composants de technologie CMOS sont perturbés de la même manière sur les deux niveaux. Cette différence de comportement selon les niveaux est attribuée à la dissymétrie de l'étage de sortie et surtout de l'étage d'entrée des composants TTL. Les étages de sortie des composants CMOS sont par contre

\section{TTL-LS $95 \mathrm{MHz}$}

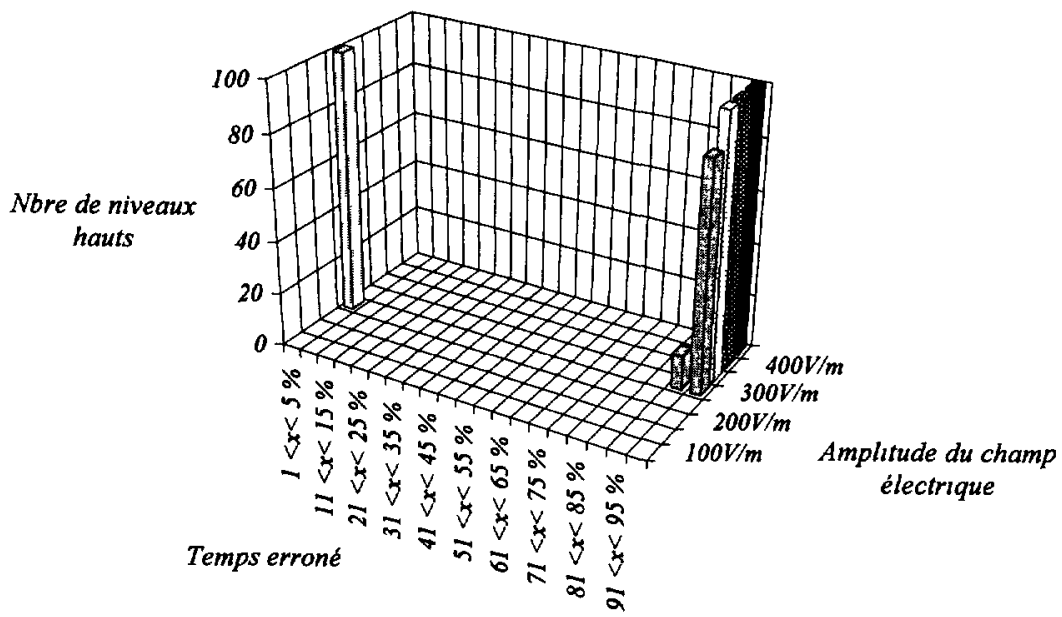

Fig. 10. - Pourcentage de temps durant lequel les niveaux hauts sont perturbés. La fréquence du perturbateur est de $95 \mathrm{MHz}$ et les composants sont de technologie TTL-LS.

[Time percent during which the high level of the signal are disturbed. The frequency of the disturbance is $95 \mathrm{MHz}$ and the components are in TTLLS technology] 


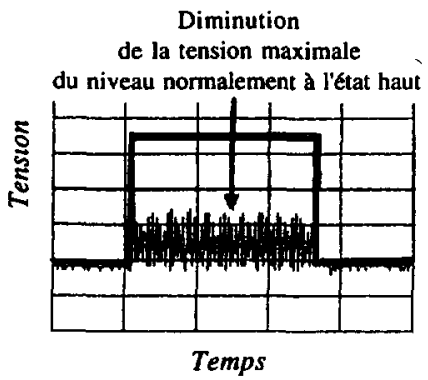

a)

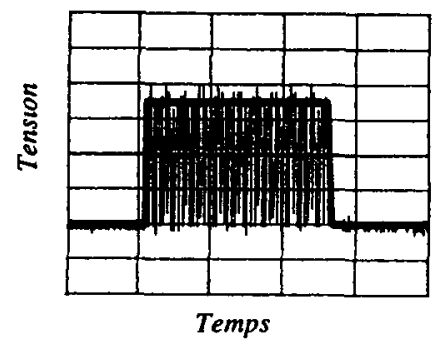

b)

Fig. 11. - Courbes temporelles obtenues pour un perturbateur de fréquence $95 \mathrm{MHz}$ et d'amplitude 450V/m. a) Technologie TTL-LS, b) Technologie TTL-AS.

[Time domain curves when the disturbance has a frequency of $95 \mathrm{MHz}$ and an amplitude of $450 \mathrm{~V} / \mathrm{m}$. a) Technology TTL-LS, b) Technology TTL-AS.]

\section{TTL-AS $95 \mathrm{MHz}$}

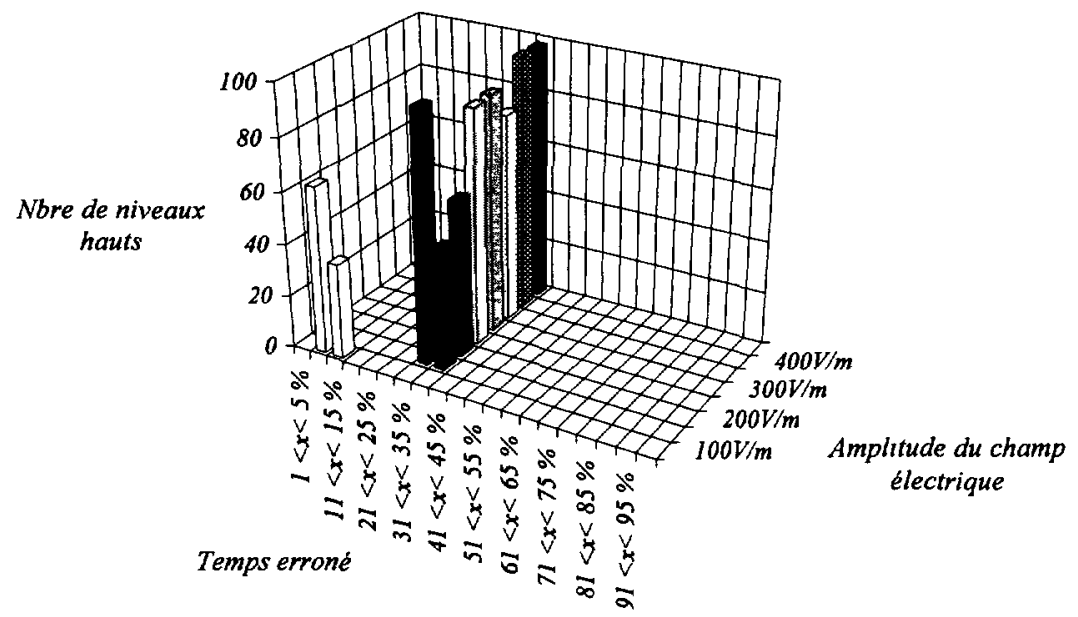

Fig. 12. - Pourcentage de temps durant lequel les niveaux hauts sont perturbés. La fréquence du perturbateur est de $95 \mathrm{MHz}$ et les composants sont de technologie TTL-AS.

[Time percent during which the high level of the signal are disturbed. The frequency of the disturbance is $95 \mathrm{MHz}$ and the components are in TTL-AS technology.]

symétriques, ce phénomène n'apparaît donc pas [9]. La technologie CMOS affiche globalement une susceptibilité électromagnétique inférieure à la technologie TTL.

Au sein de la technologie TTL, les familles ALS et LS d'une part, et les familles F et AS d'autre part, ont des comportements similaires. A $95 \mathrm{MHz}$, nous constatons qu'à partir d'une amplitude de $300 \mathrm{~V} / \mathrm{m}$, les niveaux hauts des familles LS et ALS sont totalement perturbés (Fig. 10). Ce résultat statistique est dû à une baisse de la tension maximale de sortie (Fig. 11a). La tension maximale de sortie des composants des familles AS et F ne baissant pas (Fig. 11b), le pourcentage de temps pendant lequel les niveaux hauts sont perturbés plafonne à $41 \%$ (famille AS) ou $55 \%$ (famille F) (Fig. 12). 
On retrouve cette similitude de comportement pour les technologies TTL-LS et TTL-ALS d'une part et TTL-F et TTL-AS d'autre part sur les résultats présentés dans la référence [7] (pp. 86-87). Les fonctions d'entrée/sortie des portes sont visualisées lorsqu'un signal perturbateur à la fréquence de $300 \mathrm{MHz}$ est injecté en entrée des portes. On constate un décalage vers une tension plus élevée de la tension niveau bas pour les technologies TTL-F et TTL-AS contrairement aux résultats enregistrés sur les technologies TTL-LS et TTL-ALS. La baisse de la tension maximale de sortie observée pour les technologies TTL-LS et TTL-ALS s'explique par une diminution de la tension de commutation. A la fréquence de $300 \mathrm{MHz}$, la tension de commutation diminue aussi pour les technologies TTL-AS et TTL-F. De même, pour ces deux technologies, nous avons observé sur notre dispositif sous test une baisse de la tension maximale correspondant à la présence de niveaux hauts lorsque nous augmentons la fréquence du perturbateur. Cette diminution de la tension maximale des niveaux hauts ou cette baisse de la tension de commutation des composants est en relation directe avec les paramètres suivants : fréquence du perturbateur et fréquence maximale de travail des composants. Les constructeurs fournissent une information concernant le temps de propagation des portes élémentaires telles les portes NAND ou inverseuses. On peut en déduire les fréquences maximales de travail des portes inverseuses. Elle sont respectivement de $33 \mathrm{MHz}, 45 \mathrm{MHz}, 83 \mathrm{MHz}$ et $100 \mathrm{MHz}$ pour les technologies TTL-LS, TTL-ALS, TTL-F et TTL-AS. Pour chaque famille, la diminution de la tension maximale apparaît en sortie pour des fréquences de perturbateurs supérieures à la fréquence maximale de travail des composants. Le classement des familles selon l'ordre croissant de la fréquence minimale nécessaire pour observer une diminution de tension est le suivant : TTL-LS, TTL-ALS, TTL-F et TTL-AS. Il est identique au classement des familles selon l'ordre croissant de leur fréquence maximale de travail. La diminution de la tension maximale de sortie correspondant normalement à un état háut est donc bien due à la détection-filtrage des signaux redressés en entrée. De même, les perturbations observées en sortie sur les niveaux normalement à l'état bas sont déterminées en partie par cette détection-filtrage des signaux [10].

Notons enfin que la famille "S" n'est perturbée que pour des couples fréquences / amplitudes bien déterminés, et que les résultats obtenus par l'étude statistique et les graphes visualisant l'excursion des défauts concordent $[10,11]$. Le comportement singulier de la famille "S" montre que des tests CEM réalisés uniquement pour une amplitude maximale de perturbation ne sont pas suffisants.

\section{Application à l'étude d'une carte électronique complexe}

2.1. In'TRODUCTION. - Une technique pour assurer la sûreté de fonctionnement de systèmes électriques consiste à doubler ou multiplier les voies de traitement. Les calculs de fiabilité montrent en effet que la probabilité de dysfonctionnement contraire à la sécurité d'un système diminue lorsque l'on utilise le principe de redondance. Ces calculs de probabilité considèrent que les défaillances subies par l'une et l'autre des voies n'auront pas les mêmes caractéristiques (modes de pannes, fréquence d'apparition...). Cette hypothèse n'est plus valide dès lors que des défaillances de cause commune apparaissent. L'exemple classique de cause commune est une coupure de l'alimentation des deux voies. Une autre cause possible est une perturbation due à un couplage entre le système redondant et une onde électromagnétique. Pour éviter des pannes de mode commun classiques (pannes de cause commune dont les effets sont identiques sur les deux voies), des différences de conception électronique ont été apportées par les concepteurs entre les deux voies d'une carte électronique assurant une fonction de sécurité dans un système anti-survitesse de métro automatique. Une des voies utilise des composants de technologie TTL et est alimentée en $5 \mathrm{~V}$. L'autre voie utilise des composants de technologie CMOS et est alimentée en $12 \mathrm{~V}$. En ce qui concerne les pannes dues à une perturbation électromagnétique, 


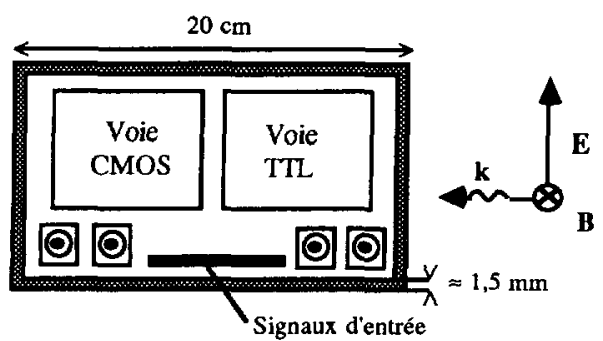

a)

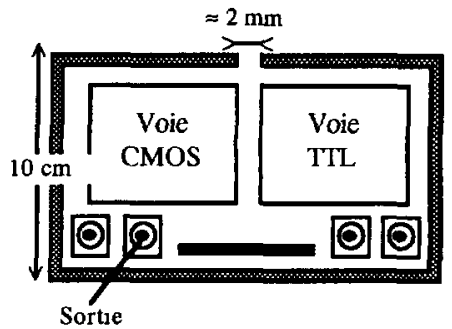

b)

Fig. 13. - a) La bande de cuivre qui délimite la carte est fermée, b) la bande de cuivre qui délimite la carte est coupée.

(a) The track which delimits the printing circuit board is normal, b) The track which delimits the printing circuit board is cut.]

l'utilisation de technologies différentes peut s'avérer être une bonne mesure pour éviter que les mêmes effets se produisent sur les deux voies. En effet, le paragraphe précédent a montré une grande différence de comportement des composants de technologie TTL et des composants de technologie CMOS lorsque qu'ils subissent un couplage électromagnétique.

L'objectif de ce paragraphe est d'essayer de vérifier sur un exemple concret si l'utilisation de composants de technologies différentes sur les voies d'un système redondant soumis à des perturbations électromagnétiques peut effectivement améliorer sa sûreté de fonctionnement. Pour mener à bien cette étude, nous avons reproduit la carte en laboratoire d'après le schéma électrique du constructeur. Quelques modifications quant à la génération des horloges sont apportées et la carte imprimée n'est pas identique. Si l'étude fonctionnelle de la carte reproduite est représentative de la carte d'origine, l'étude de sa susceptibilité par rayonnement ne l'est pas puisque le couplage diffère de part la carte imprimée. Mais elle permet de tirer certaines conclusions d'ordre général sur les systèmes redondants, et en particulier sur la carte d'origine.

Une bande de cuivre flottante est utilisée pour délimiter la carte. Lors de cette étude, nous regardons aussi l'impact de cette bande sur le couplage. Pour cela, l'étude de la susceptibilité est effectuée lorsque la bande est coupée ou non (Fig. 13).

2.2. Principe de la sÉcurité AnTi-SuRvitesse et Fonction de la CaRTE. - La sécurité anti-survitesse sur un métro est souvent assurée grâce à une ligne constituée de deux fils parallèles périodiquement croisés [12]. Cette ligne est alimentée par une onde porteuse dans la gamme des $100 \mathrm{kHz}$. Les variations de phases engendrées par les croisements de fils sont captées par une antenne magnétique située sous le véhicule. Le principe de la sécurité anti-survitesse consiste à générer un signal d'alarme si un intercroisement est parcouru en moins de $\Delta t_{1}$ dans le cas nominal et en moins de $\Delta t_{2}$ en cas de consigne de vitesse affine $\left(\Delta t_{1}<\Delta t_{2}\right)$. La vitesse du métro est donc asservie par la distance qui sépare deux croisements consécutifs.

La carte Élaboration Retard Survitesse (ERS) que nous avons étudiée a pour fonction de générer des impulsions retardées de $0,27 \mathrm{~s}$ et $0,3 \mathrm{~s}$ par rapport à la détection d'un croisement. Si un autre croisement est détecté avant leur génération, la vitesse du métro est trop rapide et une consigne de freinage d'urgence doit être impérativement générée.

2.3. Étude de la PERTURbation PAR RAYONNEMENT DE LA CARTE. - La carte ERS est placée sous une cellule "stripline" afin d'étudier sa susceptibilité à un champ électromagnétique perturbateur harmonique dont la fréquence varie entre $1 \mathrm{MHz}$ et $225 \mathrm{MHz}$. Pour éviter une 


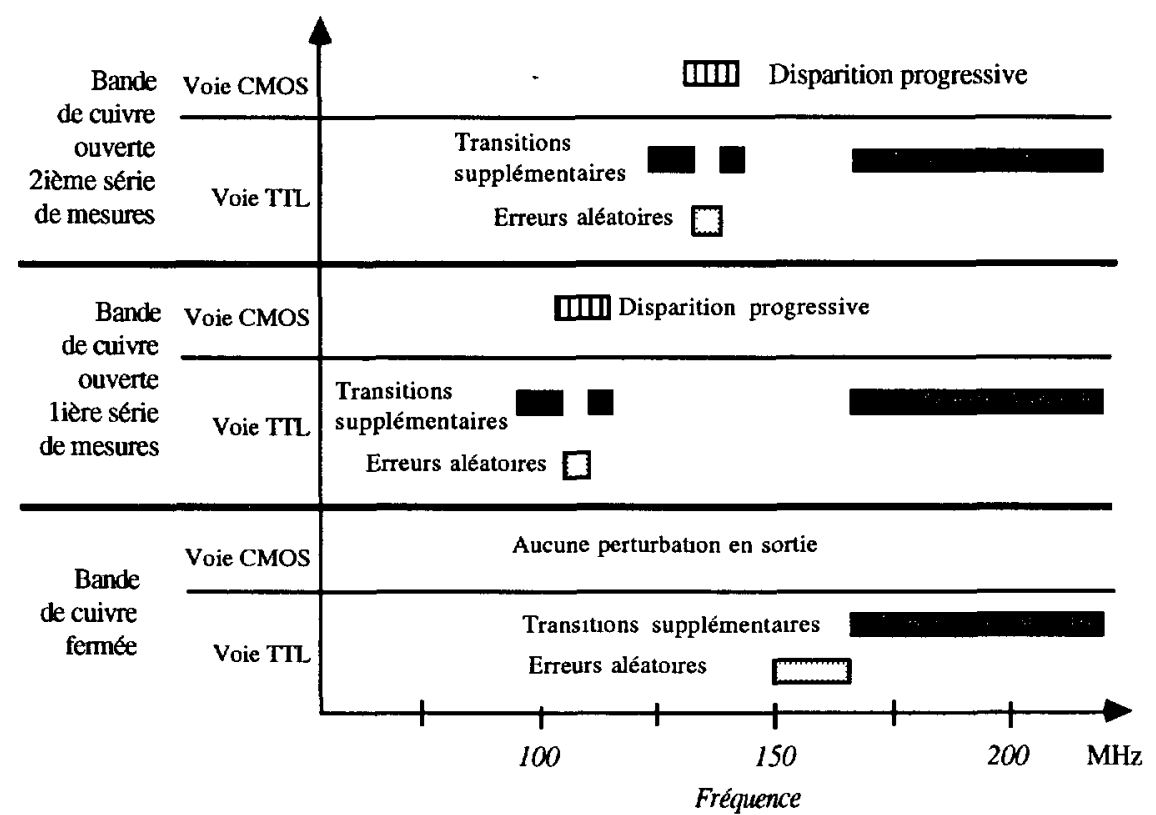

Fig. 14. - Fréquences et types de défaillances observés sur la carte ERS.

[Frequencies and types of failures observed on the printing circuit board.]

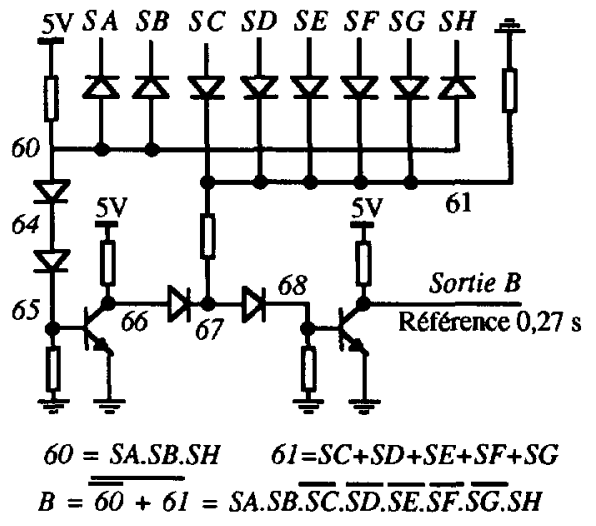

a)

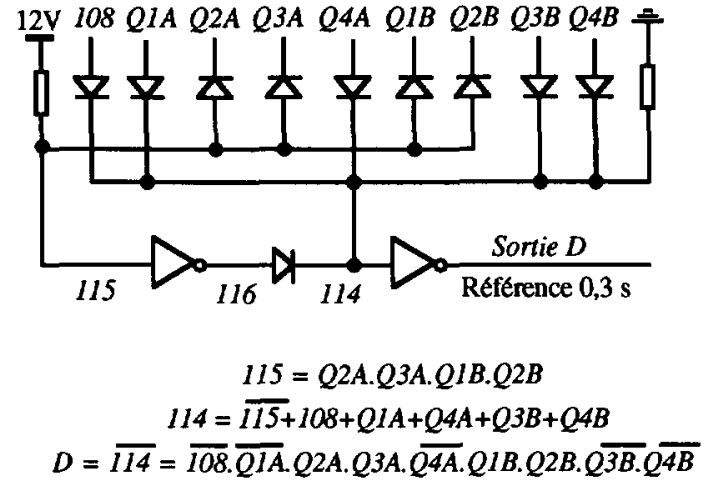

b)

Fig. 15. - Schémas électriques des étages de sortie de la carte ERS. a) Voie TTL, b) Voie CMOS. [Electrical schemes of the output stages of the printed circuit board. a) TTL channel, b) CMOS channel.]

perturbation par rayonnement des signaux d'entrée et de sortie, de la fibre optique est utilisée. Les signaux de sortie sont captés à l'aide de sondes optiques compatibles aux niveaux de la carte et réalisées en tentant de minimiser la perturbation apportée par la prise d'informations. Que la bande de cuivre qui délimite la carte ERS soit coupée ou non (Fig. 13), aucune défaillance de mode commun n'a été observée (Fig. 14). Les différences de conception entre les deux voies de la carte et notamment l'utilisation de technologies différentes vont donc dans le sens d'une plus grande sécurité de la carte. Par contre, comme la voie TTL est plus sensible aux perturbations 
rayonnées que la voie CMOS, la disponibilité de la carte serait plus grande si la voie TTL n'était pas utilisée et si la voie CMOS était dédoublée.

Nous constatons que la bande de cuivre qui délimite les cartes électroniques a un impact non négligeable sur le couplage puisque lorsque celle-ci est coupée, la susceptibilité de la carte change. Un glissement des fréquences de perturbation a été observé lorsque la bande est coupée (Fig. 14). Ce glissement est sans doute lié à une légère modification des caractéristiques de la bande de cuivre due aux nombreuses manipulations effectuées sur la carte ERS. En effet, la coupure de la bande introduit une capacité en série avec l'inductance de la bande. Une légère modification de la largeur de la coupure modifie la valeur de cette capacité ainsi que la fréquence de résonance de la boucle.

En ce qui concerne la voie TTL, on observe des erreurs aléatoires de type avance, retard ou disparition de l'impulsion et des transitions parasites supplémentaires. Ces transitions arrivent toujours au même moment par rapport à l'impulsion retardée. Elles sont dues au fonctionnement propre de la carte, à l'utilisation de transistors bipolaires pour réaliser les inverseurs de l'étage de sortie (Fig. 15a) et à l'impédance de charge présentée par notre sonde optique. En absence de sonde optique, nous avons pu montrer que ces transitions supplémentaires n'apparaissent pas $[10,11]$. En ce qui concerne la voie CMOS, seule une disparition progressive de l'impulsion est observée lorsque la bande de cuivre est coupée (Fig. 14). Des composants intégrés sont utilisés pour réaliser les inverseurs de sortie (Fig. 15b) et aucune transition supplémentaire n'est observée même en présence de la sonde optique.

\section{Conclusion}

L'étude d'un dispositif sous test simple a montré que la susceptibilité électromagnétique de circuits à base de composants logiques dépend de la technologie de fabrication de ces composants. Des similitudes ou des différences de comportement des familles de technologie sont observées. Pour les systèmes redondants, l'utilisation sur chaque voie de technologies dont les comportements sont très différents peut éviter l'apparition de pannes de mode commun dues à une perturbation électromagnétique. Il est ainsi possible d'augmenter la sécurité des systèmes. Cependant, en utilisant une technologie plus sensible sur l'une des voies, on diminue la disponibilité du système. Selon que le concepteur veut favoriser la sécurité ou la disponibilité d'un système redondant, il choisira ou non d'utiliser des technologies différentes.

Le concepteur peut aussi conserver la technologie la moins susceptible sur les deux voies et jouer sur la topologie des circuits pour tenter de diminuer la probabilité d'apparition de pannes de mode commun, dues à une perturbation électromagnétique. Cette alternative permettrait d'améliorer la sécurité et la disponibilité.

Nous pensons que le critère de susceptibilité électromagnétique doit intervenir dès la conception dans le choix d'une technologie de composants, afin de contribuer à éviter des surcoûts dus à une mise aux normes CEM tardive dans le processus de fabrication.

\section{Remerciements}

Ce travail est effectué dans le cadre du Groupement Régional Nord-Pas de Calais pour la Recherche sur les Transports (GRRT). 


\section{Bibliographie}

[1] Tront J. G., Predicting URF Upset of MOSFET Digital IC's., IEEE Trans. Electromagn. Compat. EMC-27 (1985) 64-69.

[2] Laurin J. J., Zaky S. G. et Balmain K. G., EMI induced delays in digital circuits : Prediction., IEEE 1992 Symposium on Electromagnetic Compatibillity (Anaheim CA August 1992) pp. 443448.

[3] Chappel J. F. et Zaky S. G., EMI induced delays in digital circuits : Application., IEEE 1992 Symposium on Electromagnetic Compatibillity (Anaheim CA August 1992) pp. 449-454.

[4] Coudoro B., Étude phénoménologique de la sensibilité des circuits logiques fonctionnant en régime dynamique soumis aux effets induits par des champs électromagnétiques., Thèse de Doctorat $\mathrm{N}$ 1193 USTL, (1993).

[5] Larde C., Étude expérimentale du comportement des circuits intégrés logiques soumis à des perturbations électromagnétiques., Thèse de Doctorat N 834 USTLFA (1991).

[6] Heddebaut B., Étude phénoménologique et modélisation du comportement de fonctions logiques élémentaires TTL et CMOS soumises à des perturbations induites par couplages électromagnétiques., Thèse de Doctorat N 972 USTL (1992).

[7] Klingler M., Étude phénoménologique de la sensibilité électromagnétique de composants électroniques logiques implantés sur circuits imprimés, Thèse de Doctorat N 988 USTL (1992).

[8] Larson C.E. et Roe J.M., A Modified Ebers-Moll Transistor Model For RF-Interference Analysis., IEEE Trans. Electromagn. Compat. EMC-21 (1979) 283-290.

[9] Maréchal C., Klingler M., Szelag M. et Heddebaut M., Analyse et étude statistique du comportement de composants logiques soumis à une perturbation sinusoïdale., Symposium ITTG 93, Innovation Technologique dans les Transports Guidés (Lille Septembre 1993) pp. 407-422.

[10] Maréchal C., Étude de l'influence de la technologie et de l'association de composants logiques sur la sensibilité électromagnétique de cartes électroniques. Application à l'étude d'une fonction dont la sécurité est fondée sur la redondance matérielle., Thèse de Doctorat N 1380 USTL (1994).

[11] Maréchal C., Étude de l'impact d'une perturbation électromagnétique sur le fonctionnement de composants logiques électroniques, Deuxième conférence des jeunes chercheurs en génie électrique (Grenoble, Avril 1994) pp. I-15-I-18.

[12] Heddebaut M., Influence des perturbations électromagnétiques sur le fonctionnement de cartes sécuritaires de dispositifs de conduite automatisée de métros, 4ème colloque et exposition sur la CEM Juin 1987, Session H-1. 
Articles Réguliers

Regular Papers 